\title{
Pain mechanisms
}

\author{
H.W. Kosterlitz \\ Unit for Research on Addictive Drugs, Marischal College, University of Aberdeen, \\ Aberdeen AB9 1AS UK.
}

\section{Introduction}

When I was asked to chair jointly with Professor J.S. Han a Symposium on "Pain Mechanisms", I was reminded of a Research Report Lars Terenius and I arranged for a Dahlem Konferenz entitled "Pain and Society" which had to examine strategies in the study of pain and its role in society. The report was the result of a discussion held in Berlin in 1979 (Kosterlitz $\&$ Terenius, 1980). The main themes were the evolution of expression of acute and chronic pain, the central mechanisms of pain control, mechanisms and models of recurrent persistent pain and finally, the principles of pain management. It is interesting to note that five of the contributors to today's symposium were then deeply involved with these problems; the sixth contributor is Professor Han who will report on pharmacological studies of acupuncture analgesia; we are particularly glad that he agreed to take part in this symposium.

Since time immemorial, pain has been the outstanding symptom which persuades the patient to seek the advice of his doctor. Until a few decades ago, the only effective treatment of acute and chronic pain was the administration of analgesic drugs. Since then we have acquired a much better understanding of the anatomical and physiological bases of pain which have made possible a number of new therapeutic approaches to this difficult problem. We are also becoming more aware of the psychological and social factors which influence a person's attitude and reaction to pain whether it be of short or long duration.

Probably the most significant progress in recent years has been the discovery of the role of certain neuropeptides in the transmission and modulation of the impulses which signal noxious changes and are appreciated as pain. One of these neuropeptides, substance $P$, seems to facilitate this transmission in the periphery while some of the opioid peptides appear to exert an inhibitory modulation and in this manner limit the experience of pain to tolerable levels. We now have to realize that the situation is very complex. We have three large opioid peptides, pro-opiocortin, pro-enkephalin and pro-dynorphin from which are derived smaller active peptides. Some of these fragments are $\beta$-endorphin derived from pro-opiocortin, [Met]enkephalin, metorphamide or adrenorphin and [Leu]enkephalin from proenkephalin, and, finally, $\alpha$ - and $\beta$ - neoendorphin and dynorphin $\mathrm{A}$ and dynorphin $\mathrm{B}$ from pro-dynorphin; they have affinities to two or three $\mu, \delta$ - and $x$-binding sites. Our understanding of these opioid fragments is still limited but we hope to hear more of this problem in the presentations which follow.

The topic concerning pain is multidisciplinary in a wide sense. For a long time philosophers have been engaged in trying to achieve an understanding of the metaphysical meaning of pain. In recent times behavioural scientists have become deeply involved in the problems of pain as they affect the individual and society. Different approaches have been employed by neuroanatomists and neurophysiologists to elucidate the role which different parts of the central nervous system play in the experience and appreciation of different types of pain; neurochemists and particularly neuropharmacologists have aimed at alleviation of pain by analysing the events at a cellular level and study in their modification by drugs.

Both Dr Han and I hope that the discussion presented in this symposium will increase our understanding of the important phenomenon of pain and help in alleviating the suffering it may cause.

\section{Reference}

KOSTERLITZ, H.W. \& TERENIUS, L.Y. (1980). Introduction. In Pain and Society. Kosterlitz, H.W. \& Terenius, L.Y. (eds) pp. 1-2, Dahlem Konferenzen 1980, Weinheim, Verlag Chemie GmbH. 